\title{
A NEW FREQUENCY METHOD APPLIED TO CALCULATE CABLE FORCE FOR CABLES WITH THE INTERMEDIATE MULTI-SUPPORT
}

\author{
Pingjie Li Ronghui Wang Niujing Ma
}

School of Civil Engineering and Transportation, South China University of Technology(pingjieli@163.com)

\begin{abstract}
This paper proposes a new frequency method applied to calculate cable force for cables with the intermediate multi-support from measured natural frequencies. The proposed method has established new relationship between cable frequency and tension force, taking cable force, the intermediate multi-support, flexural rigidity, boundary conditions of two ends into account. Following the classical guidelines of cable dynamics, and by making some simplifications, frequency equation has been obtained in non-dimensional form. When natural frequencies have measured, cable force can be calculated by solving frequency equation. Comparison with Matharmethod and the proposed method in the field test, the difference of cable force result between two methods is just only 1\%, so the proposed method has been made to validate.
\end{abstract}

Keywords:frequency method, cable force, multi-support, cable

\section{INTRODUCTION}

Frequency method is the way to calculate the cable force by measured natural frequencies of cables. In recent years, Frequency method has received increasing attention because of its simplicity and speediness. In practice, frequency method is widely used for cables with boundary conditions of constraints on both ends, and rarely used for cables with boundary conditions of constraints on both ends and the intermediate. As cables are applied more and more widely, the boundary condition of cables is more and more complex, such as the damper at suspenders in the suspension bridge and intermediate support at the tie bar in the arch bridge. So a new frequency method is useful for application to calculate the tension force of cables with the intermediate multi-support.

Nowadays, there are a lot of researches on the vibration of cables. Irvine $\mathrm{H} \mathrm{M}^{[1]}$ has proposed an analytical solution for the frequency equation for sagged cable without bending stiffness. For such long cables, with such large diameters, flexural stiffness cannot be neglected in determining dynamical properties of cables. So Zui $\mathrm{H}$ ${ }^{[2]}$,Geier Rand Kim B H ${ }^{[3-4]}$ has proposed methods which allow us to obtain cable force in a simple manner, which are based on the identification of modal properties of cables. Flamand $\mathrm{O}$ and Matsumoto $\mathrm{M}^{[5-6]}$ have observed the influence of rain-wind action to cables. These studies are mostly for cables with the boundary condition of constraints on both ends, and there are rare for cables with the boundary condition of 
constraints on both ends and the intermediate. $\mathrm{R} \mathrm{H}$ wang ${ }^{[7]}$ has put forward to a finite element solution for tension force of cables with elastic multi-support, which has been experimentally verified. However, there is not an analytic method for tension force of cables with elastic multi-support.

This paper proposes a new frequency method for application to calculate the tension force of cables with the intermediate multi-support from measured natural frequencies. The proposed method has established new relationship between cable frequency and tension force, taking cable force, the intermediate multi-support, flexural rigidity, boundary conditions of two ends into account. Following the classical guidelines of cable dynamics, and by making some simplifications, frequency equation has been obtained in non-dimensional form. When natural frequencies have measured, cable force can be calculated by solving frequency equation.

\section{DYNAMIC MODEL OF CABLES}

\subsection{Vibration model for cables}

The cable with the boundary condition of restraints on both ends and the intermediate is equivalent for the multi-span beam with axial force, when flexural stiffness of cables has not been neglected., the calculated parameters can be described as show in figure 1: $\mathrm{L}$ is the undeformed cable length; $\mathrm{H}$ is the horizontal component of initial cable tension; $\mathrm{n}$ is the number of constraints on the intermediat of cables; $\mathrm{K}_{\mathrm{i}}(\mathrm{i}=1,2, \ldots ., \mathrm{n})$ is stiffness of constraints; $\mathrm{X}_{\mathrm{i}}(\mathrm{i}=1,2, \ldots, n)$ is the distance between the adjacent constraints. The boundary condition of constraints on both ends is consider as clamped.

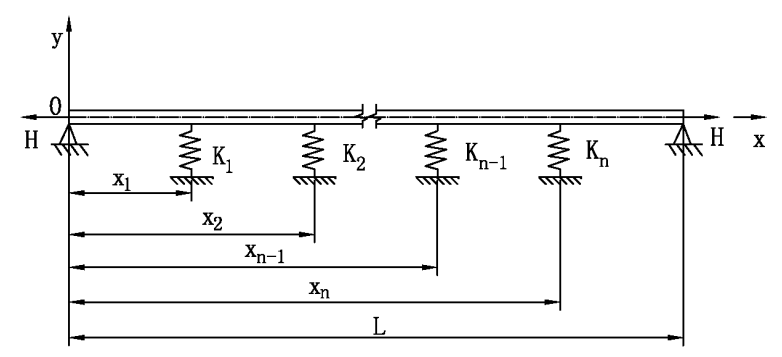

Figure 1. the cable with the intermediate multi-support

In accordance with ${ }^{[1]}$, the linearized equations of motion are:

$$
H \frac{\partial^{2} v}{\partial x^{2}}+h(t) \frac{d^{2} y}{d x^{2}}-E I \frac{\partial^{4} v}{\partial x^{4}}-\mu_{y} \dot{v}+f_{y}(x, t)=m \ddot{v}
$$

$v$ is the transverse vibration displacement of cables changing over time, $h(t)$ is the additional horizontal component of tension changing over time, $y(x, t)$ is the transverse coordinate of cables, $\mathrm{x}$ is the coordinate along the axis of beam, $\mathrm{t}$ is 
vibration time, $\mathrm{E}$ is the Young modulus; I is the inertia moment; $\mu_{y}$ is viscous damping coefficients per unitary cable length, $\dot{v}$ is first time derivative of the vibration displacement, $\ddot{v}$ is second time derivative of the vibration displacement, $\mathrm{m}$ is the cable mass per unitary length, $f_{y}(x, t)$ is impressed force changing over time.

The vibration model of the cable has considered elastic support only, but hasn't considered the effect of damping, so $\mu_{y} \dot{v}=0$.according to Irvine $\mathrm{H} \mathrm{M}^{[1]}$, It can be negligible to the additional horizontal component of tension ${ }^{[1]}$, so $h(t)=0$.

Assuming $\mathrm{v}(\mathrm{x}, \mathrm{t})=\varphi(\mathrm{x}) q(t), \quad f_{y}(x, t)=f_{y}(x) q(t)$, the partial differential equation (1) is transformed into two ordinary differential equations, one governing the time evolution and one governing the spatial distribution of the motion:

$$
\begin{aligned}
& \ddot{q}(t)+\omega^{2} q(t)=0 \\
& \varphi^{I V}(x)-\frac{H}{E I} \varphi^{\prime \prime}(x)-\frac{m \omega^{2}}{E I} \varphi(x)=\frac{1}{E I} f_{y}(x)
\end{aligned}
$$

Expression (2b) is the fourth order nonhomogeneous linear differential equation, which can be solved as:

$$
\varphi(x)=A_{1} \cos (\alpha x)+A_{2} \sin (\alpha x)+A_{3} \cosh (\beta x)+A_{4} \sinh (\beta x)-\frac{1}{m \omega^{2}} f_{y}(x)
$$

In which $\mathrm{Aj}(\mathrm{j}=1, \ldots, 4)$ are constants and the following quantities have been introduced:

$$
\begin{aligned}
& \alpha(\omega)=\sqrt{\sqrt{\left(\frac{H}{2 E I}\right)^{2}+\omega^{2} \frac{m}{E I}}-\frac{H}{2 E I}} \\
& \beta(\omega)=\sqrt{\sqrt{\left(\frac{H}{2 E I}\right)^{2}+\omega^{2} \frac{m}{E I}}+\frac{H}{2 E I}}
\end{aligned}
$$

\subsection{The cable frequency equation}

Boundary conditions for clamped restraint at cable anchorages are:

$$
\begin{aligned}
& v(-L / 2, t)=0 \\
& v(L / 2, t)=0 \\
& \frac{\partial v}{\partial x}(-L / 2, t)=0 \\
& \frac{\partial v}{\partial x}(L / 2, t)=0
\end{aligned}
$$

The boundary conditions of cables with clamped ends, given by Eqs. (5a)- (5d), 
substitute into Eq (3); And then, an algebraic set of four equations, with the unknown quantities $\operatorname{Bj}(\mathrm{j}=1, \ldots, 4)$, can be solved; And $\mathrm{Bj}(\mathrm{j}=1, \ldots, 4)$ can be substituted into Eq. (3) and the final expression of the modal shapes is obtained following:

$$
\hat{\varphi}(\hat{x})=\frac{\hat{f}_{y}(x)}{\hat{\omega}^{2}}\left\{1-\frac{\hat{\alpha} \cosh (\hat{\beta} \hat{x}) \sin (\hat{\alpha} / 2)+\hat{\beta} \cos (\hat{\alpha} \hat{x}) \sinh (\hat{\beta} / 2)}{\hat{\alpha} \cosh (\hat{\beta} / 2) \sin (\hat{\alpha} / 2)+\hat{\beta} \cos (\hat{\alpha} / 2) \sinh (\hat{\beta} / 2)}\right\}
$$

The parameters of expression (6) can be obtained by Eqs. (7a)- (7f). $\delta\left(x-x_{i}\right)$ is the Dirac delta function.

$$
\begin{aligned}
& \hat{x}=\frac{x}{L} \\
& \hat{\omega}=\omega \frac{L^{2}}{\sqrt{E I / m}} \\
& \xi=L \sqrt{H / E I} \\
& \hat{\alpha}(\hat{\omega})=\sqrt{\sqrt{\left(\frac{\xi^{2}}{2}\right)^{2}+\hat{\omega}^{2}}-\frac{\xi^{2}}{2}} \\
& \hat{\beta}(\hat{\omega})=\sqrt{\sqrt{\left(\frac{\xi^{2}}{2}\right)^{2}+\hat{\omega}^{2}}+\frac{\xi^{2}}{2}} \\
& \hat{f_{y}}(\hat{x})=\frac{K_{i} L^{4}}{E I} \sum_{i=1}^{n} \hat{\varphi}\left(\hat{x}_{i}\right) \delta\left(\hat{x}-\hat{x}_{i}\right) \quad(i=1,2, \ldots ., n)
\end{aligned}
$$

So frequency equation of cables can be gotten by Eq (6) and Eq (7f), the simplified expressions is written as:

$$
|C+\eta I|=0
$$

The parameters of expression (8) can be gotten by Eqs. (9a)- (9b)

$$
\begin{gathered}
\eta_{i}=-\frac{E I \hat{\omega}^{2}}{K_{i} L^{4}}(i=1,2, \ldots ., n) \\
C_{j i}=1-\frac{\hat{\alpha} \cosh \left(\hat{\beta} \hat{x}_{i}\right) \sin (\hat{\alpha} / 2)+\hat{\beta} \cos \left(\hat{\alpha} \hat{x}_{i}\right) \sinh (\hat{\beta} / 2)}{\hat{\alpha} \cosh (\hat{\beta} / 2) \sin (\hat{\alpha} / 2)+\hat{\beta} \cos (\hat{\alpha} / 2) \sinh (\hat{\beta} / 2)} \quad(j=1,2, \ldots ., n \quad i=1,2, \ldots ., n)
\end{gathered}
$$

\section{CABLE FORCE CALCULATION}

\subsection{Cable force equation}

Cable frequency equation (8) contains two non-dimensional parameters, one is 
the non-dimensional frequency $\hat{\omega}$, and the other is the non-dimensional cable force $\xi$. So cable force equation can be obtained by eqs (8), (7d), (7e):

$$
f(\hat{\omega}, \xi)=0
$$

As cable frequency is tested out, the non-dimensional frequency $\hat{\omega}$ can be gotten. So expression (10) is a single parameter equation as show in expression (11).

$$
f(\xi)=0
$$

\subsection{Iterative algorithm for the cable force equation}

As we known, the $\mathrm{Eq}(11)$ is a transcendental equation. It can't be solve directly. So it must adopt numerical methods, like Nowton-Raphson, for the purpose of obtaining the roots of Eq. (11).It is convenient to manipulate this expression to eliminate some differences, which, when they are made between close approximate numbers, can lead to round off errors as show in figure 2.

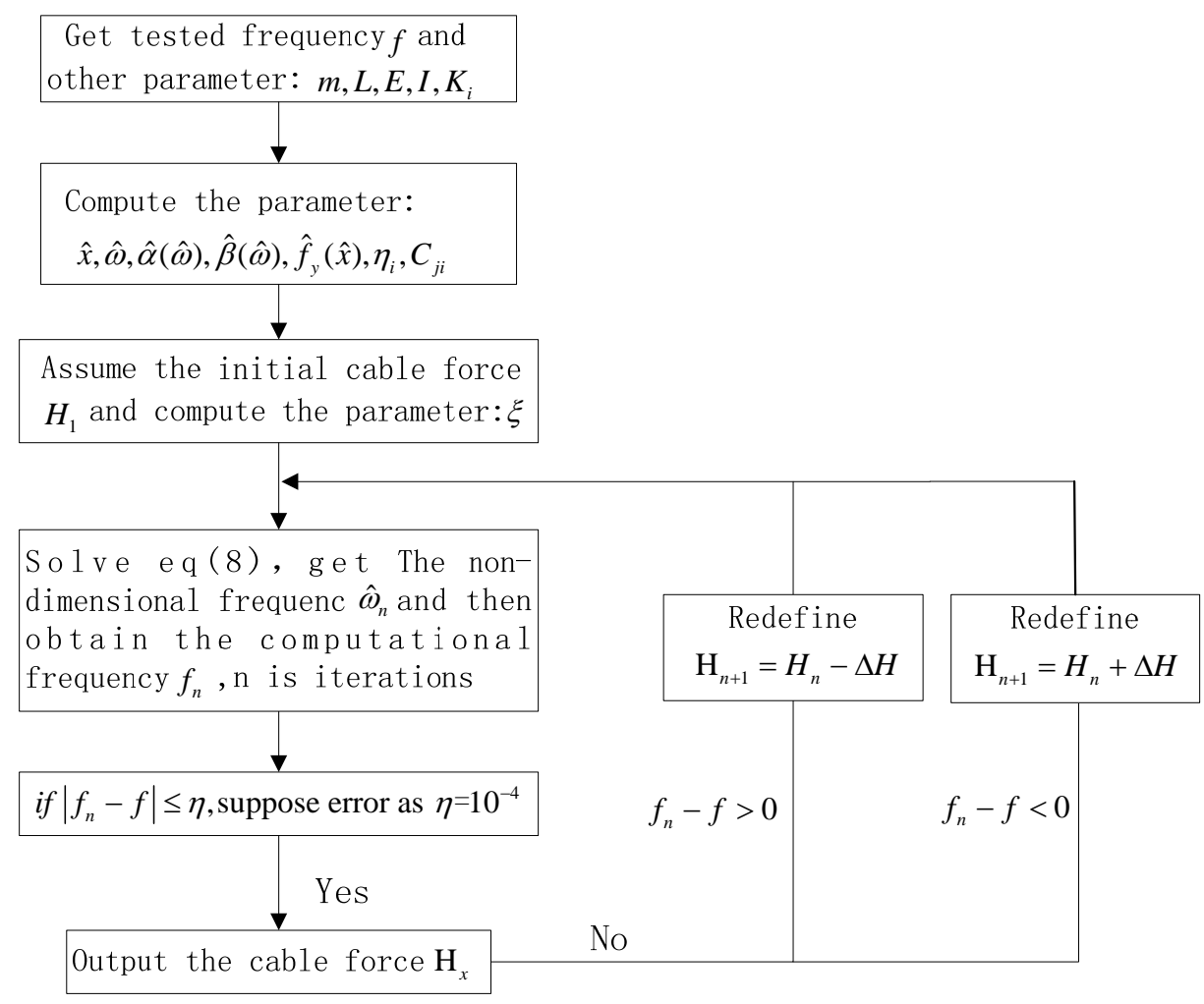

Figure 2. flowsheet for cable force calculation

\section{APPLICATION EXAMPLE}

\section{1 background}


Sanshanxi Bridge is concrete filled steel tubular tied arch bridge. Tie bar, consisting of hundreds of steel strands, is located on the both end of bridge under the bridge deck with the length of $260 \mathrm{~m}$, which is used for resisting horizontal thrust of main arch. A bunch of tie includes 10 to 12 steel strands, and these steel strands are contact each other. There are 50 bunches of tie separated. Steel strands have been separated to three parts by points silk plate. The length of parts of steel strands is $49.7 \mathrm{~m}, 160.6 \mathrm{~m}, 49.7 \mathrm{~m}$ respectively. Points silk plate is welding inside steel box, which is using for protecting the tie bar from corrosion. Steel strand and points silk plate contact tightly, so each bunch of tie bar can be considered to be anchored at the place of points silk plate. It can't be completed to determine the axial force of tie bar by applying frequency method directly if we have not solved the following two problems. One is how to test frequency of tie bar. Steel strands are contact each other, so we can apply some filling blocks to separate the steel strand, what's important is determine the compression stiffness of filling blocks. The other is how to calculate the axial force of tie bar by measured frequency. There is changed boundary condition of steel strand by increased filling blocks. So it's essential to deduce a new equation of cable force and frequency for cables with the intermediate multi-support.

\subsection{Test parameter}

Tie bar and steel strand both can be consider to be the cable, but it just can be test one steel strand frequency, so the cable is stand for steel strand in the following. The cable parameter is describing at table $1, \mathrm{E}$ is the modulus of elasticity, $\mathrm{I}$ is the inertia moment, $\rho$ is the mass density, A is area, $\mathrm{d}$ is diameter of section, $\mathrm{L}$ is the length of cables.

Table 1. test parameter

\begin{tabular}{cccccc}
\hline $\mathrm{E}(\mathrm{pa})$ & $\mathrm{I}\left(\mathrm{m}^{4}\right)$ & $\rho\left(\mathrm{kg} / \mathrm{m}^{3}\right)$ & $\mathrm{A}\left(\mathrm{m}^{2}\right)$ & $\mathrm{d}(\mathrm{mm})$ & $\mathrm{L}(\mathrm{m})$ \\
& & & & \\
\hline $1.95 \mathrm{E}+11$ & $9.69 \mathrm{E}-10$ & $7.85 \mathrm{E}+3$ & 0.000137 & 15.2 & 49.7 \\
\hline
\end{tabular}

The filling block has three layers. There is steel plate at the top and bottom layer with the thickness of $5 \mathrm{~mm}$ and block rubber at the middle layer with the thickness of $40 \mathrm{~mm}$. The size of filling blocks is $5 \mathrm{~cm} \times 5 \mathrm{~cm}$. The compression stiffness of filling blocks is determined by triaxial compression test. Two filling blocks have been made and the compression stiffness of them are respectly : $K_{1}=1.0464 \times 10^{6} \mathrm{~N} / \mathrm{m}$ 、 $\mathrm{K}_{2}=1.0104 \times 10^{6} \mathrm{~N} / \mathrm{m}$.

\subsection{Cable frequency and cable force}

Cable frequency cannot be tested directly because of the contact between cables, so filling blocks are used for isolating cables as show in figure 3. And cable frequency 
can be tested by dynamic testing instrument when optimum vibration system set at the middle of two filling blocks as show in figure 4, which are installed under the cable as show in figure 5.

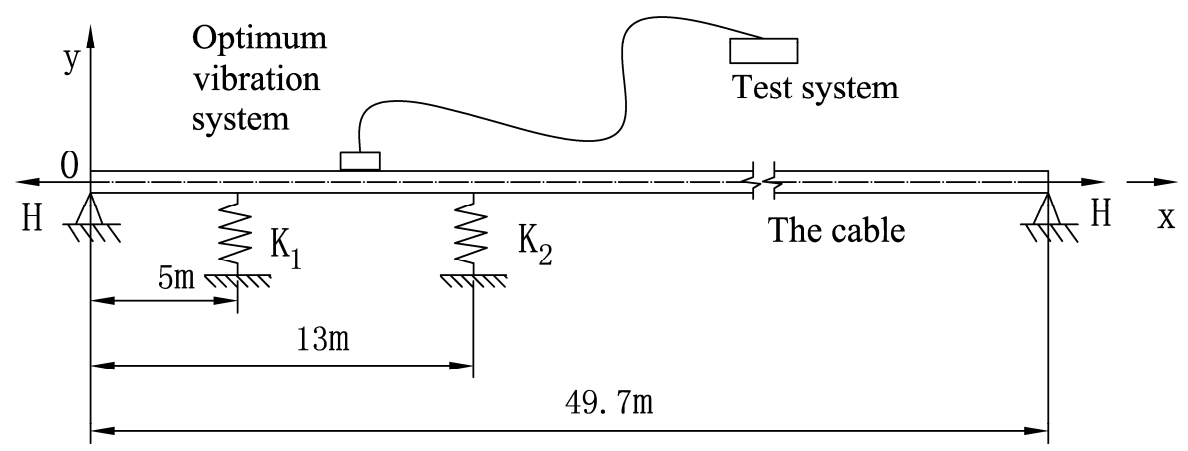

Figure 3. The model for testing cable frequency

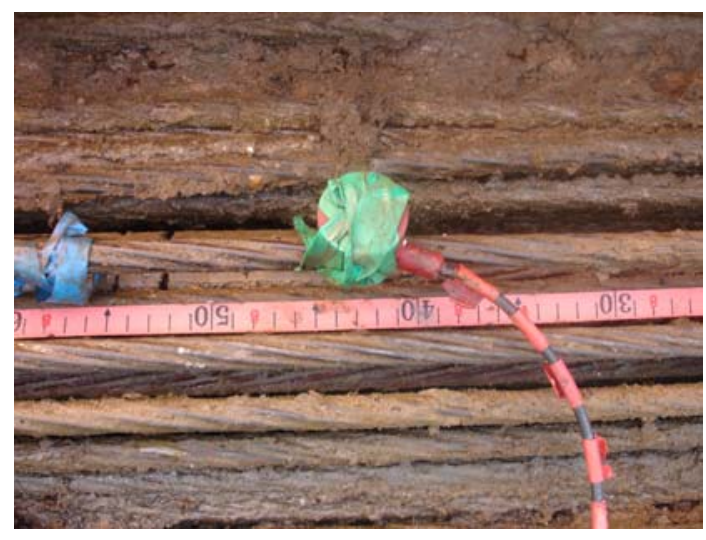

Figure 4. The installing place of optimum vibration system

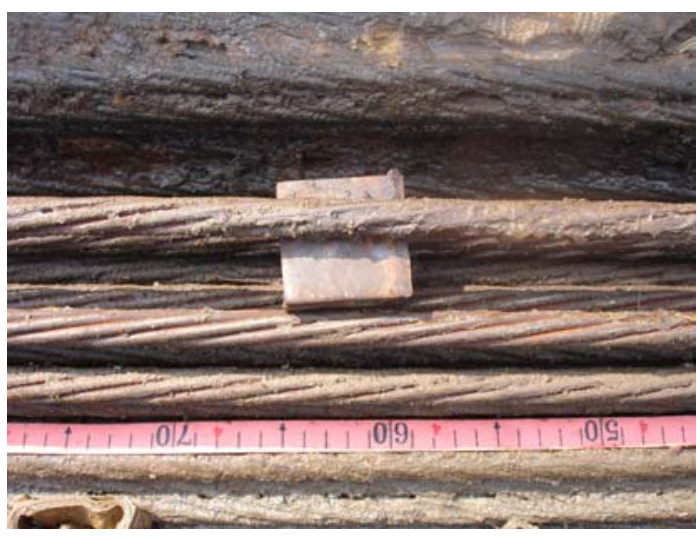

Figure 5. The installing place of the filling block

It's find that the 4th order frequency of cables can be easy tested as show in table 2.

Table 2. Tested cable frequency

\begin{tabular}{cccccc}
\hline number & 1 & 2 & 3 & 4 & 5 \\
\hline The 4th order cable frequency $(\mathrm{Hz})$ & 16.2109 & 17.041 & 16.3086 & 16.2598 & 16.3574 \\
\hline
\end{tabular}

By flowsheet for cable force calculation (fig.2), cable force has calculated as show in table 3 . The average cable force is $97.4 \mathrm{kN}$, which can evaluate the whole level of tie bar force. Using non-destructive testing (NDT) methods of Matharmethod ${ }^{[8]}$, tie bar force can be tested to be $98.3 \mathrm{kN}$. So the difference of tie bar force tested by two methods is small, just with the error of $1 \%$.

Table 3. Calculated cable force

\begin{tabular}{ccccccc}
\hline number & 1 & 2 & 3 & 4 & 5 & average \\
\hline cable force $(\mathrm{kN})$ & 94.55 & 105.01 & 95.75 & 95.15 & 96.36 & 97.4 \\
\hline
\end{tabular}




\section{CONCLUSION}

The computational formula of frequency method cannot calculate cable force for cables with boundary conditions of constraints on the intermediate. so a new computational formula of frequency method is established for cables with the intermediate multi-support. Some conclusions are made as following:

1) The proposal computational formula can be useful for application to calculate the tension force of cables with the intermediate multi-support, such as the damper at suspenders in the suspension bridge and intermediate support beam at the tie bar in the arch bridge.

2 ) The proposal computational formula is a transcendental equation, so numerical method should be adopted, but it's easy to make a programme, following fig 2 ,to calculating cable force.

3) The field test shows that the difference of cable force, testing by the proposal method and the matharmethod, is small, so the proposal method is reliable.

\section{Acknowledgement}

This work is supported by Program of National Natural Science Foundation of China (50978105)

\section{REFERENCE}

[1]Irvine H M. Cable Structures [M]. Cambrige:The MIT Press, 1981.

[2] Zui H, Shinke T, Namita Y. Practical formulas for estimation of cable tension by vibration method [J]. J Struct Eng 1996, 122(6):651-665.

[3] Geier R, De Roeck G, Flesch R. Accurate cable force determination using ambient vibration measurements. Struct Infrastruct Eng 2006;2(1):43-52.

[4] Kim B H, Park T. Estimation of cable tension force using the frequency-based system identification method [J]. Journal of Sound and Vibration. 2007, 304(3-5): 660-676.

[5] Flamand O. Rain-wind induced vibration of cables. J Wind Eng Ind Aerodyn 1995;57:353-62.

[6] Matsumoto M, Daito Y, Kanamura T, Shigemura Y, Sakuma S,Ishizaki H. Wind-induced vibration of cables of cable-stayed bridges.J of Wind Eng and Ind Aerodyn 1998;74-76:1015-27.

[7]Wang Rong-hui,Gan Quan,Gong Ling-ling,et al.A finite element solution for tension of cable with elastic multi-support[J]. Journal of Vibration Engineering, 2010, 23(5):567-571.

[8]BA50/93. Post-tensioned Concrete Bridges. Planning, Organisation and Methods for Carrying out Special Inspections. Department of Transport. July 1993. 